\title{
Outcomes of a randomised controlled trial of a complex genetic counselling intervention to improve family communication
}

\author{
Jan Hodgson ${ }^{\star, 1,2}$, Sylvia Metcalfe ${ }^{1,2}$, Clara Gaff ${ }^{2,3,4}$, Susan Donath ${ }^{2,5}$, Martin B Delatycki ${ }^{6,7}$, Ingrid Winship ${ }^{4,8}$, \\ Loane Skene ${ }^{9}$, MaryAnne Aitken ${ }^{3,10}$ and Jane Halliday ${ }^{2,11}$
}

\begin{abstract}
When an inherited genetic condition is diagnosed in an individual it has implications for other family members. Privacy legislation and ethical considerations can restrict health professionals from communicating directly with other family members, and so it is frequently the responsibility of the first person in a family to receive the diagnosis (the proband) to share this news. Communication of genetic information is challenging and many at-risk family members remain unaware of important information that may be relevant to their or their children's health. We conducted a randomised controlled trial in six public hospitals to assess whether a specifically designed telephone counselling intervention improved family communication about a new genetic diagnosis. Ninety-five probands/parents of probands were recruited from genetics clinics and randomised to the intervention or control group. The primary outcome measure was the difference between the proportion of at-risk relatives who contacted genetics services for information and/or genetic testing. Audit of the family genetic file after 18 months revealed that $25.6 \%$ of intervention group relatives compared with $20.9 \%$ of control group relatives made contact with genetic services (adjusted odds ratio (OR) $1.30,95 \%$ confidence interval $0.70-2.42, P=0.40$ ). Although no major difference was detected overall between the intervention and control groups, there was more contact in the intervention group where the genetic condition conferred a high risk to offspring (adjusted OR 24.0, 95\% confidence interval 3.4-168.5, $P=0.001$ ). The increasing sophistication and scope of genetic testing makes it imperative for health professionals to consider additional ways of supporting families in communicating genetic information.
\end{abstract}

European Journal of Human Genetics (2016) 24, 356-360; doi:10.1038/ejhg.2015.122; published online 1 July 2015

\section{INTRODUCTION}

The diagnosis of a new genetic condition in an individual or his/her child is likely to have implications for other family members who may themselves be at risk of developing the condition and/or passing it on to their children. Under these circumstances successful communication is usually dependent on the ability of the newly diagnosed patient (proband), or parents of the proband, to communicate complex genetic information to their family members. Many factors have been identified that may have an impact on successful family communication about genetic risk including existing family relationship characteristics, feelings of anxiety or guilt, understanding of inheritance and/or the genetic condition and the ability of lay individuals to effectively explain complex genetic information. ${ }^{1}$ Effective family communication occurs less frequently than is optimal with some estimates suggesting that only $15-20 \%$ at-risk relatives become aware of relevant and important information. ${ }^{2-4}$ Family members usually express a desire to pass on this information themselves ${ }^{5,6}$ with support from health professionals, if necessary, to explain more complicated concepts. ${ }^{7}$ It seems likely that most relatives would intend to pass on genetic risk information and that, where this does not occur (non-disclosure), this is usually a 'passive' failure to disclose rather than the proband actively avoiding communication. ${ }^{8}$ We suggest that there is a role for health professionals in supporting probands in relation to 'passive' non-disclosure. There has been much debate about how health professionals should be involved with this process; the extent to which this is deemed important appears to vary according to the information that can be made available and the condition that is involved. ${ }^{2,9}$

Two previous Australian studies have explored how professionals might assist in family communication about genetics. One study involving letters sent from the genetics service, via the proband or directly to relatives, found that there was an increase in uptake of genetic testing by relatives from $23 \%$ in the group dependent on communication by the proband to $40 \%$ in the group receiving information directly. ${ }^{10}$ Another study utilised a specific discussion over the telephone, followed by a call $2-4$ weeks post result and another 3-6 months later if indicated. This resulted in a 2.6-fold increase in contact with services ${ }^{11}$ and provided evidence that

${ }^{1}$ Genetics Education and Health Research, Murdoch Childrens Research Institute, Royal Children's Hospital, Parkville, Victoria, Australia; ${ }^{2}$ Department of Paediatrics, University of Melbourne, Parkville, Victoria, Australia; ${ }^{3}$ Murdoch Childrens Research Institute, Royal Children's Hospital, Parkville, Victoria, Australia; ${ }^{4}$ Department of Medicine, University of Melbourne, Parkville, Victoria, Australia; ${ }^{5}$ Clinical Epidemiology and Biostatistics Unit, Murdoch Childrens Research Institute, Royal Children's Hospital, Parkville, Victoria, Australia; ${ }^{6}$ Department of Clinical Genetics, Austin Health, Heidelberg, Victoria, Australia; ${ }^{7}$ Bruce Lefroy Centre for Genetic Health Research, Murdoch Childrens Research Institute, Royal Children's Hospital, Parkville, Victoria, Australia; ${ }^{8}$ Genetic Medicine, Royal Melbourne Hospital, Parkville, Victoria, Australia; ${ }^{9}$ Melbourne Law School, University of Melbourne, Parkville, Victoria, Australia; ${ }^{10}$ Research Office, La Trobe University, Melbourne, Victoria, Australia; ${ }^{11}$ Public Heath Genetics, Murdoch Childrens Research Institute, Royal Children's Hospital, Parkville, Victoria, Australia

${ }^{*}$ Correspondence: Dr J Hodgson, Genetics Education and Health Research, Murdoch Childrens Research Institute, Royal Childrens Hospital, Parkville 3052 Victoria, Australia. Tel: +61 38341 6308; E-mail: jan.hodgson@mcri.edu.au

Received 7 January 2015; revised 30 April 2015; accepted 6 May 2015; published online 1 July 2015 
additional genetic counselling may assist probands with family communication.

The study presented here, a multicentre randomised trial, aimed to further investigate whether a specific genetic counselling intervention increased the number of relatives who were made aware of the possible risk to their own health or the health of their offspring and the availability of genetic testing.

The main hypothesis was that a specifically designed genetic counselling complex intervention, delivered by phone at up to three time points over 12 months, would facilitate communication of important genetic information within families.

\section{MATERIALS AND METHODS}

\section{Experimental design}

This was a randomised controlled trial (RCT) to assess the effect of additional genetic counselling support (the intervention) on contact with genetic services by at-risk family members. The primary outcome measure, frequency of contact, was considered a proxy for communication of genetic information between family members.

The protocol and full details of the methodology used in this study have been published separately. ${ }^{12}$ Approval for all aspects of the trial was obtained from the Human Research Ethics Committee at participating hospitals in Victoria, Australia: Southern Health, Royal Childrens Hospital, Royal Melbourne Hospital, Bendigo Health, Ballarat Health and Austin Health Service. All participants gave written informed consent before taking part in the study.

\section{Study participants—eligibility}

From 2009 to 2012, the participants were approached when they attended a genetics clinic for the first time at six public hospitals across the state of Victoria, Australia. Eligible potential participants were the first person in a family to be diagnosed, or have a child diagnosed, with a genetic condition or as a carrier of a genetic condition that has implications for other family members and for which genetic testing is possible. They needed to be able to speak, read and write English, have at least one at-risk relative who resided in Victoria and be aged 18 years or over. Those who gave consent were randomised into the intervention or control group.

\section{Recruitment at genetic clinics}

Initially the clinics participating were general genetic clinics where clients were seen for many reasons. An interim audit of the clinics over a 3-month period late in 2009 revealed that, of 516 clients seen, only 5\% were eligible for participation, with the others being seen for prenatal testing (21\%), clinical review (14\%), a condition with no family implications (16\%), a condition with no genetic diagnosis available (17\%), had multiple appointments already (18\%) or other reasons $(9 \%)$. To enhance recruitment of eligible participants, in March 2010 we added recruitment from cancer genetics clinics.

\section{Study intervention design}

The intervention needed to be clinically relevant and feasible, reproducible in practice and conforming to Victorian State privacy legislation. ${ }^{13}$ Utilising a theoretical basis consistent with genetic counselling practice, that is, nondirective and patient centred, a telephone genetic counselling intervention was specifically designed. The aim was to enhance the ability of each individual to identify and overcome existing barriers in communicating genetic information within their family.

The 'complex' nature of the intervention is critical to the study design. Complex interventions are 'built up from a number of components, which may act both independently and interdependently'. ${ }^{14}$ The function and process of a complex intervention should be standardised, but not the components themselves. ${ }^{15}$ They are particularly useful in the context of health service activities and allow for a degree of flexibility in delivery of the intervention package. Experts in the field of complex interventions state that 'strict standardisation may be inappropriate; the intervention may work better if a specified degree of adaptation to local settings is allowed for in the protocol ${ }^{16}$ p.980. We chose this pragmatic RCT design to allow for some individualisation of the genetic counselling service. Tailoring the intervention to the participant, that is, the individualisation of the number of phone calls, was acceptable because once all designated relatives had been approached, the intervention served no further purpose.

Genetic counsellors were trained to carry out the intervention and were monitored for adherence to the protocol. This process involved both group and individual training by the developers of the intervention-a senior genetic counsellor/researcher in family communication and an academic genetic counselling researcher. In addition, at least one mock intervention phone call, based on a likely scenario, was undertaken with each counsellor. These were audiotaped and evaluated to assess and address any noted divergences from the intervention protocol. ${ }^{17}$ The intervention was implemented two to three times over a 12-month period, with some flexibility according to feedback from participants about the amount of contact they already had made and a determination by the genetic counsellor as to the need for ongoing communication; that is, if all relatives had been contacted, no further phone calls were deemed necessary.

\section{Data collection}

A three-generation pedigree was obtained by the genetic counsellor at the first clinic visit for each participant. From this, the number of at-risk relatives at that point in time was derived. A person's 'at-risk status' is based on genetic relatedness, taking into account the inheritance pattern for the condition involved. Patient files for all participants were examined 18 months after recruitment to determine number of clinic contacts by relatives (see Figure 1).

\section{Analysis}

Data were entered into an ACCESS database and statistical analyses undertaken in STATA (StataCorp. 2011. Stata Statistical Software: Release 12, College Station, TX, USA: StataCorp LP). Uptake of genetic services was measured as the total number of at-risk relatives who contacted the service divided by the total number of at-risk relatives identified on pedigrees 18 months later. A relative who was initially considered to be at risk, but whose risk had

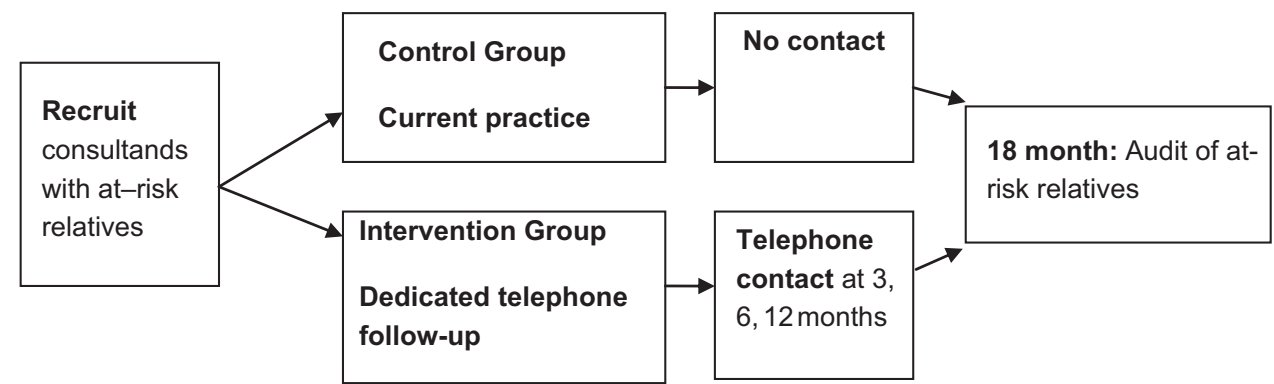

Recruitment

Randomisation

Intervention

Measurements

Figure 1 Participant flow of events. 
subsequently been modified following a negative test result from another family member, was excluded at this time.

The odds of contact by relatives of those in the intervention arm compared with the odds of contact by relatives of those in the control arm (together with 95\% confidence intervals (CIs) for the odds ratios (ORs)) were estimated using (i) univariable logistic regression (unadjusted estimates) and (ii) a marginal logistic regression model fitted using generalised estimating equations with information sandwich estimates of SE (adjusted estimates allowing for correlation between the responses of relatives of the same participants).

Families were assigned to one of three categories on the basis of the availability of an intervention to reduce disease onset or severity (actionability) and the immediacy of risk to living relatives and offspring related to the genetic information.

Category 1: Intervention available. Genetic information has implications for close relatives and there is an intervention (surveillance or treatment), for example, cancer susceptibility genes including BRCA1, BRCA2 and Lynch Syndrome, inherited cardiac conditions (for example, long QT syndrome and hypertrophic cardiomyopathy).

Category 2: High offspring risk. Genetic information has implications for close relatives and the risk to offspring is high if a relative is found to be a carrier, for example, chromosomal anomalies such as translocations, $\mathrm{X}$-linked conditions such as Fragile X syndrome and Duchenne/Becker muscular dystrophy.

Category 3: Low offspring risk. Genetic information has implications for relatives, and the risk to offspring is low if a relative is found to be the carrier, for example, autosomal recessive conditions such as cystic fibrosis, deafness and spinal muscular atrophy.

We investigated whether the effect of the intervention differed between these assigned categories by testing for an overall interaction between intervention and category. Where there was evidence of a differential effect, we analysed the subgroups separately.

The likelihood of contact in a subgroup analysis of these assigned categories, irrespective of the intervention arm, was examined to determine whether there was a differential effect on contact. All analyses were by intention to treat, that is, participants were included in the group to which they were randomly assigned, and all participants were included in the analysis (including those who did not receive all three intervention phone calls).

\section{RESULTS}

Ninety-five participants were recruited between June 2009 and January 2012. There were a number of stages in the recruitment process at which there was attrition or loss to follow-up of potential participants. These are shown in Figure 2.

Table 1 summarises the age and gender of the participants and shows the number recruited in the three categories. It also shows the same characteristics of their eligible relatives, identified in the genetic files.

The major finding of the RCT was that $25.6 \%(142 / 554)$ of the intervention group relatives made contact with genetic services, compared with $20.9 \%(112 / 536)$ of the control group relatives. The adjusted OR, taking into account the clustering effect within families, was 1.30; and the $95 \% \mathrm{CI}$ was $0.70-2.42, P=0.40$.

There was some evidence that the effect of the intervention differed between subgroups ( $P$-value for interaction, 0.02); therefore, the subgroups were analysed separately. Results for each subgroup are shown in Table 2.

In Category 2 (high offspring risk), the frequency of contact was markedly higher in the intervention arm than the control arm. The overall low frequency of contact in Category 3 did not differ between the arms of the RCT.

\section{DISCUSSION}

This RCT set out to discover whether a specifically designed genetic counselling intervention, delivered by telephone at up to three time points after a new genetic diagnosis, would increase family communication. While there was no significant difference overall between the intervention and control groups, there were some differences observed between subgroups, on the basis of the degree of risk to the relatives and the ability to intervene to reduce risk of disease onset or severity.

For Category 1 conditions, where there are appropriate treatments or active surveillance, the level of family communication was highest ( $\sim 30 \%$ overall); this group is most likely to have received encouragement from their health professionals to pass on information. The lack of difference between the intervention and control groups is likely to reflect the urgency with which both groups of participants recognise the need to impart the genetic information to their immediate relatives, who may themselves be at risk.

For Category 3 participants, where the risk to offspring is low, the intervention had no influence on the overall low contact rate. This result may indicate that knowledge of the carrier status about recessive conditions is not a strong trigger for talking to relatives, even after discussion with a genetic counsellor. The low contact rate overall may also reflect that this category included participants who were new parents, identified as carriers through newborn screening. They were either coping with a newborn affected child or were not concerned as they had been told that their child was healthy.

Where the intervention did make a difference in the level of contact with genetics services between the intervention and control group was for the Category 2 (high offspring risk) participants. For this group of participants it seems likely that the intervention phone calls successfully assisted them in identifying family members with a high risk of passing on the condition to their offspring and provided support and guidance in disclosing the genetic information.

The findings in this study are all in relation to Mendelian conditions, such as cystic fibrosis, fragile $\mathrm{X}$ syndrome, inherited cancer, long QT syndrome and SMA, as well as balanced translocation carriers. Clearly, family awareness of these conditions is important and discussion about this is invariably included as part of the genetic counselling session supplied by the genetics service providers, the counsellors imparting the genetic information routinely to the client. These findings suggest that it may be particularly effective to offer targeted assistance for families where a new genetic diagnosis confers a low risk to existing family members but a high risk to future offspring.

For some conditions, it is recognised that disclosure of risk information is absolutely essential and relying on proband dissemination may be inappropriate. To address this, guidelines concerning disclosure of genetic information by Australian health professionals were developed in 2009 to protect health professionals who inform genetic relatives in cases where there has been active non-disclosure by the client. ${ }^{18}$ Typical scenarios are presented in the guidelines to demonstrate how best to deal with such problematic situations and have in common a potential harm to genetic relatives who are not informed of an at-risk status. The conditions used in the scenarios in this privacy guideline were polycystic kidney disease, breast cancer, Huntington disease, familial adenomatous polyposis, balanced chromosome translocation carrier, fragile $\mathrm{X}$ and diabetes. The guidelines address questions such as: what factors support the need for disclosure or weigh against disclosure; what information could be given to a patient (genetic relative) by the authorised representative; who might be involved in decision making and how might disclosure take place? The development of these guidelines took several years, multiple consultations with clinicians and community and brought to 


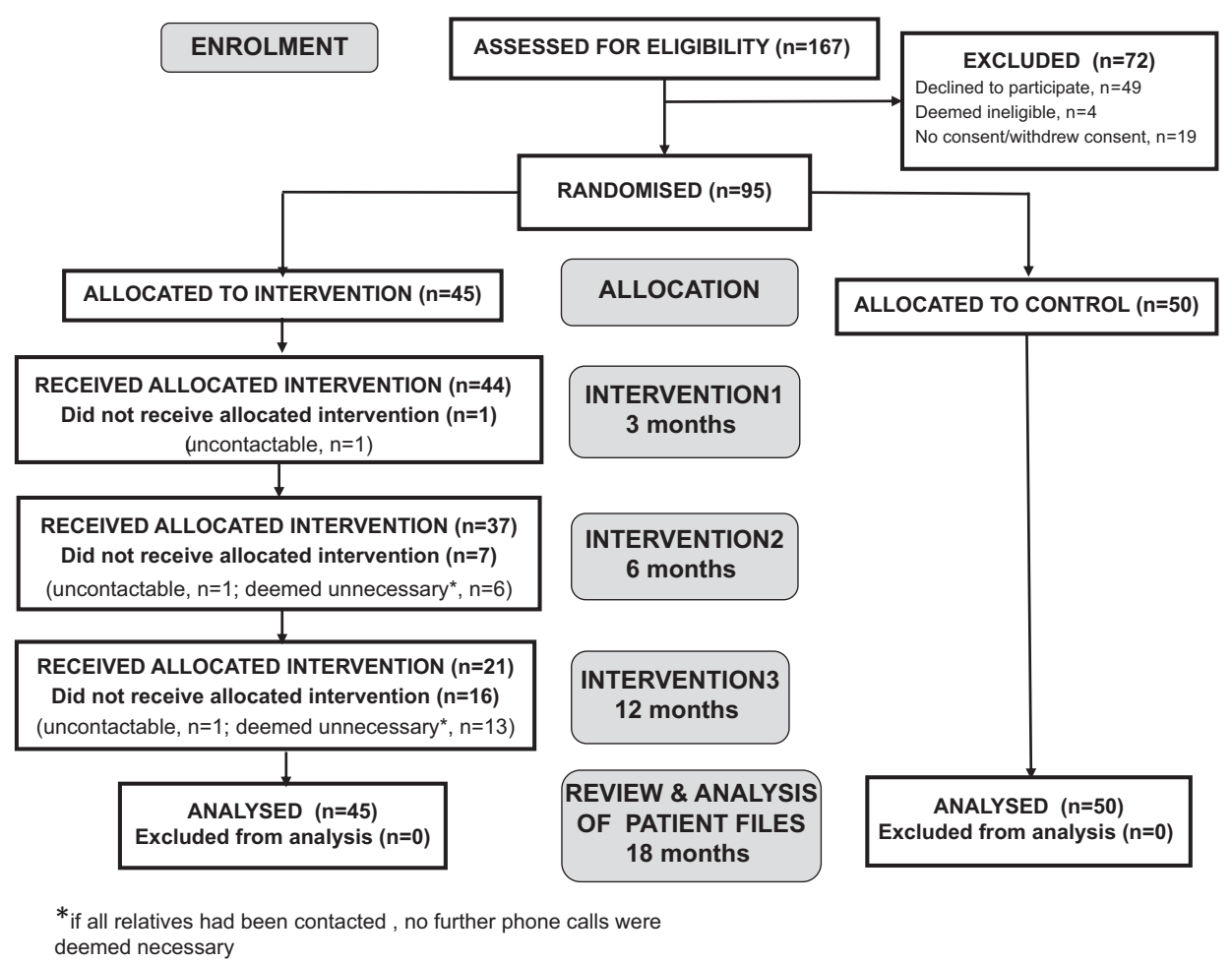

Figure 2 Flow of participants through the trial.

Table 1 Characteristics of trial participants

\begin{tabular}{lcc}
\hline Number of participants & Intervention $\mathrm{n}=45$ & Control $\mathrm{n}=50$ \\
\hline Age of participant (years) mean (s.d.) & $49.5(14.9)$ & $45.8(13.9)$ \\
Gender of participants, female \% & 86.7 & 88.0 \\
& & \\
Participant category, $\mathrm{n}$ (\%) & & $25(50.0)$ \\
Intervention available & $46(57.8)$ & $5(10.0)$ \\
High offspring risk & $15(33.3)$ & $20(40.0)$ \\
Low offspring risk & 554 & 536 \\
Total number of at-risk ${ }^{\text {a relatives }}$ & $12.3(8.5)$ & $10.7(7.6)$ \\
Average number per participant, mean(s.d.) & 49.5 & 48.3 \\
Gender of at-risk relatives, female \% & & $304(56.7)$ \\
& & $60(11.2)$ \\
Number of at-risk relatives by category (\%) & $19(3.4)$ & $172(32.1)$ \\
Intervention available & $179(32.3)$ & \\
High offspring risk & & \\
Low offspring risk &
\end{tabular}

aA person's 'at-risk status' is on the basis of genetic relatedness, taking into account the inheritance pattern for the condition involved.

Table 2 Subgroup analysis of frequency of contact with Victorian genetic services and likelihood of contact by the intervention group compared with the control group as reference for each category

\begin{tabular}{lccccc}
\hline \multicolumn{5}{c}{ Frequency of contact, \% (n) } \\
Category & Intervention & Control & Adjusted OR (95\% Cl) & P-value \\
\hline Intervention available & $30.3(108)$ & $28.0(85)$ & $1.12(0.50-2.50)$ & 0.28 \\
High offspring risk & $63.2(12)$ & $6.7(4)$ & $24.0(3.42-168.47)$ & 0.001 \\
Low offspring risk & $12.3(22)$ & $13.4(23)$ & $0.91(0.35-2.37)$ & 0.84 \\
\hline
\end{tabular}

the fore many concerns that people have about this issue, although it is stated clearly up front that situations in which such disclosure by the health professional may be required are rare. ${ }^{18}$

Although communication of information about genetic conditions within families is seen as a central component of genetic consultations, ${ }^{19}$ the interim audit of over 500 routine consultations at general genetics clinics, not the specialized cancer or cardiac clinics, found that only $5 \%$ were being seen for reasons that warranted disclosure of genetic information to family members. Therefore, in the professional experience of a genetic health professional, the magnitude of the need for clients attending general genetics clinics to be counselled about disclosure of genetic information is small. This stands in contrast to specialist genetic clinics for dominant, adult onset conditions such as hereditary cancer.

Genome sequencing, occurring increasingly frequently in clinical settings, presents new challenges for family communication about genetic risk. There is now much debate about the type of genetic information that should be disclosed by the health professional to the client and a growing number of guidelines and commentaries cover this topic of primary disclosure. ${ }^{20,21}$

We do not yet know what is likely to happen in a number of possible future testing scenarios regarding family communication of genetic information-for example, in the case of disclosure to parents of a child who has exome sequencing about the discovery of a pathogenic mutation or an incidental finding of potential relevance. Disclosure of genetic information will also now be warranted in families where an individual has chosen to have genome sequencing performed by direct to consumer companies. Communication of increasingly uncertain and complex genetic information is likely to present additional challenges to family communication. It is for all of these reasons that additional support from health professionals is likely to be needed and appreciated. 


\section{Limitations}

The eventual sample size, resulting from challenges with recruitment, was lower than originally anticipated and may explain the main result, which, although demonstrating a positive trend was not significant. The study was not powered to undertake the subgroup analyses that did, however, reveal a strong association in Category 2, albeit with a very wide $\mathrm{CI}$.

Across both the intervention and control groups there were mostly low levels of contact by family members with genetics services. It is important to note that the outcome measure used in this RCT, attendance at a Victorian genetic clinic, is a proxy measure that was used because it would be clearly unethical to ask family members directly whether they had received new genetic information from the participant. In addition, family members may have been informed but chosen not to access the Victorian genetic services directly or at that particular time point. It is also possible that they attended genetics services outside the state of Victoria, accessed information from other health professionals such as their general practitioner or searched online for web-based resources. Therefore, it is very likely that the findings here represent an underestimation of the actual level of family communication that has taken place.

A RCT methodology has obvious limitations, and it is important to consider just how effectively a RCT can evaluate a complex intervention such as we have described here. ${ }^{22} \mathrm{~A}$ recent review of literature in this area identified several major challenges including the standardisation of interventions, differences in approaches by those carrying out interventions and determining appropriate evaluation measures. ${ }^{23}$ Since this study began, there have been additional suggestions about how to develop effective family communication tools using the Theory of Planned Behaviour, ${ }^{24}$ although these are yet to be evaluated.

\section{Conclusions and policy implications}

The pragmatic study design utilised here, taking place within a realworld setting and congruent with genetic counselling principles, has allowed us to demonstrate that a genetic counselling intervention to enhance family communication can be successfully imparted across several settings and conditions. Although we were unable to detect major differences between all groups with regard to family communication, our findings have shown that family communication about genetic conditions that confer a high risk to offspring can be enhanced by additional professional support. As the scope and availability of genetic testing increases, it is vital that health providers and health policy makers prioritise development of evidence-based interventions that can be individually tailored. This will enable support to be offered to an increasing number of individuals who will be in possession of genetic information that needs to be communicated to family members.

\section{CONFLICT OF INTEREST}

The authors declare no conflict of interest.

\section{ACKNOWLEDGEMENTS}

We are grateful to all of the research participants and the genetic counsellors for their role in this project. We thank Hannah Brown, Veronica Collins, Denise Goodwin, Phuong Nguyen, Jean Paul and Loren Plunkett for their roles within the project team. This study was funded by grant from the National Health and Medical Research Council (NHMRC), Project No 491214. This research was supported by the Victorian Government's Operational Infrastructure Support Program.

\section{AUTHOR CONTRIBUTIONS}

All authors have made a substantial contribution to the conception and design of the study and participated in regular steering committee meetings throughout the trial. J Hodgson and J Halliday co-authored the first draft of this paper and all of the authors participated in further drafting and editing.

\section{DISCLAIMER}

The manuscript contains original material which is not under review elsewhere. The study received appropriate ethical review at all of the research sites.

1 Wilson BJ, Forrest K, van Teijlingen ER et al: Family communication about genetic risk: the little that is known. Commun Genet 2004; 7: 15-24.

2 Gaff CL, Clarke AJ, Atkinson P et al: Process and outcome in communication of genetic information within families: a systematic review. Eur J Hum Genet 2007; 15: 999-1011.

3 Landsbergen K, Verhaak C, Kraaimaat F, Hoogerbrugge N: Genetic uptake in BRCAmutation families is related to emotional and behavioral communication characteristics of index patients. Fam Cancer 2005; 4: 115-119.

4 Clayton EW: What should the law say about disclosure of genetic information to relatives? J Health Care Law Policy 1998; 1: 373-390.

5 Claes E, Evers-Kiebooms G, Boogaerts A, Decruyenaere M, Denayer L, Legius E: Communication with close and distant relatives in the context of genetic testing for hereditary breast and ovarian cancer in cancer patients. Am J Med Genet A 2003; 116A: 11-19.

6 Kerzin-Storrar L, Wright C, Williamson PR et al: Comparison of genetic services with and without genetic registers: access and attitudes to genetic counselling services among relatives of genetic clinic patients. J Med Genet 2002; 39: e85.

7 McConkie-Rosell A, Finucane B, Cronister A, Abrams L, Bennett R, Pettersen B: Genetic counseling for fragile $X$ syndrome: updated recommendations of the National Society of Genetic Counselors. J. Genet Couns 2005; 14: 249-270.

8 Gaff C, Collins V, Symes T, Halliday J: Facilitating family communication about predictive genetic testing: probands' perceptions. J Genet Couns 2005; 14: 133-140.

9 Forrest LE, Delatycki MB, Skene L, Aitken M: Communicating genetic information in families - a review of guidelines and position papers. Eur J Hum Genet 2007; 15: 612-618.

10 Suthers GK, Armstrong J, McCormack J, Trott D: Letting the family know: balancing ethics and effectiveness when notifying relatives about genetic testing for a familial disorder. J Med Genet 2006; 43: 665-670.

11 Forrest LE, Burke J, Bacic S, Amor DJ: Increased genetic counseling support improves communication of genetic information in families. Genet Med 2008; 10: 167-172.

12 Hodgson JM, Metcalfe SA, Aitken M et al: Improving family communication after a new genetic diagnosis: a randomised controlled trial of a genetic counselling intervention. BMC Med Genet 2014; 15: 33.

13 Hodgson J, Gaff C: Enhancing family communication about genetics: ethical and professional dilemmas. J. Genet Couns 2013 Feb; 22: 16-21.

14 Medical Research Council. A framework for development and evaluation of RCTs for complex interventions to improve health, 2000, p.2

15 Hawe P, Shiell A, Riley T: Complex interventions: how "out of control" can a randomised controlled trial be? Br Med J 2004; 328: 1561-1563.

16 Craig $P$, Dieppe $P$, Macintyre $S$ et al: Developing and evaluating complex interventions: the new Medical Research Council guidance. Br Med J 2008; 337: 979-983.

17 Gaff C, Hodgson J: A genetic counseling intervention to facilitate family communication about inherited conditions. J. Genet Couns 2014; 23: 814-823.

18 National Health and Medical Research Council (NHMRC): Use and disclosure of genetic information to a patient's genetic relatives under Section 95AA of the Privacy Act 1988 (Cth), 2014; https://www.nhmrc.gov.au/guidelines-publications/pr3.

19 Gaff C, Galvin K, Bylund C: Facilitating family communication about genetics in practice; in Gaff C, Bylund C (eds): Family Communication about Genetics: Theory and Practice. New York: Oxford University Press, 2010.

20 Green RC, Berg JS, Grody WW et al: ACMG recommendations for reporting of incidental findings in clinical exome and genome sequencing. Genet Med 2013; 15: 565-574.

21 van El CG, Cornel MC, Borry $\mathrm{P}$ et al: Whole-genome sequencing in health care: recommendations of the European Society of Human Genetics. Eur J Hum Genet 2013; 21: 580-584.

22 Sanson-Fisher RW, Bonevski B, Green LW, D'Este C: Limitations of the randomized controlled trial in evaluating population-based health interventions. Am J Prev Med 2007; 33: 155-161.

23 Datta J, Petticrew M: Challenges to evaluating complex interventions: a content analysis of published papers. BMC Public Health 2013; 13: 568.

24 Wiens $\mathrm{M}$, Wilson $\mathrm{B}$, Honeywell $\mathrm{C}$, Etchegary $\mathrm{H}$ : A family genetic risk communication framework: guiding tool development in genetics health services. J Commun Genet 2013; 4: 233-242. 